Bull. Korean Math. Soc. 47 (2010), No. 5, pp. 883-888

DOI 10.4134/BKMS.2010.47.5.883

\title{
ON ARMENDARIZ IDEALS
}

\section{Sh. Ghalandarzadeh, H. Haj Seyyed Javadi, M. Khoramdel,} AND M. SHAMSADDINI FARD

\begin{abstract}
In this paper, we introduce the concepts of Armendariz ideals and abelian ideals and record some results involving them.
\end{abstract}

\section{Introduction}

Throughout this paper, all rings are associative with identity. In [7] M. B. Rege and S. Chhawchharia introduced the notion of an Armendariz ring. They defined a ring $R$ to be an Armendariz ring if whenever polynomials $f(x)=$ $\sum_{i=0}^{m} a_{i} x^{i}, g(x)=\sum_{j=0}^{n} b_{j} x^{j} \in R[x]$ satisfy $f(x) g(x)=0$, then $a_{i} b_{j}=0$ for all $i$ and $j$ (The converse is always true). The term of an Armendariz ring was chosen because E. Armendariz [2, Lemma 1] had noted that a reduced ring satisfies this condition. In this paper we study Armendariz ideals; this concept is related to that of Armendariz rings.

\section{Armendariz ideals}

In this section we define and study Armendariz and abelian (one-sided) ideals. All our left-sided concepts and results have right-sided counterparts. The right annihilator of a subset $A$ of a ring $R$ is denoted by $r_{R}(A)$ or $r(A)$ (when $R$ is clear from the context). We begin with the following definition.

Definition 2.1. Let $R$ be a ring.

(a) A left ideal $I$ of $R$ is called Armendariz if whenever polynomials $f(x)=$ $\sum_{i=0}^{m} a_{i} x^{i}, g(x)=\sum_{j=0}^{n} b_{j} x^{j} \in R[x]$ satisfy $f(x) g(x) \in r_{R[x]}(I[x])$ we have $a_{i} b_{j} \in r_{R}(I)$ for all $i, j$.

(b) A left ideal $I$ of $R$ is called abelian if for each idempotent element $e \in$ $R, e r-r e \in r_{R}(I)$ for any $r \in R$.

Remark. (1) In case of a two-sided ideal the terms 'Armendariz (abelian) on the left' and 'Armendariz (abelian) on the right' will be used to avoid confusion.

(2) Trivially, the zero ideal is always both Armendariz as well as abelian (on the left as well as on the right).

Received August 21, 2007; Revised August 24, 2009.

2000 Mathematics Subject Classification. 16D25, 16N99, 16U20.

Key words and phrases. Armendariz ideal, IFP ideal. 
(3) The ideal $R$ of a ring $R$ is Armendariz (resp., abelian) on the left or on the right in the sense of the above definition if and only if the ring $R$ is Armendariz (resp., abelian).

(4) Clearly, the converse assertion of the condition of Definition 2.1(a) holds for every ring $R$ and every left ideal $I$ of $R$ since $\left.r_{R[x]}(I[x])=r_{R}(I)[x]\right)$.

(5) In the literature the term 'Armendariz' has also been used in the context of rings which may not have an identity. It is hoped that the terms 'Armendariz left ideal' and 'abelian left ideal' introduced by us in this paper will not cause confusion.

Proposition 2.2. Let $R$ be a ring and $I$ be an Armendariz left ideal of $R$. If $f_{1}, f_{2}, \ldots, f_{n} \in R[x]$ are such that $f_{1} f_{2} \cdots f_{n} \in r_{R[x]}(I[x])$, then $a_{1} \cdots a_{n} \in$ $r_{R}(I)$ where $a_{i}$ is a coefficient of $f_{i}$.

Proof. We employ the method used in the proof of Proposition 1 of [1]. Let $f_{1} \cdots f_{n} \in r_{R[x]}(I[x])$ and let $a_{i}$ be any coefficient of $f_{i}$. Now we have $f_{1}\left(f_{2} \cdots f_{n}\right) \in r_{R[x]}(I[x])$, so $a_{1} b \in r_{R}(I)$ for any coefficient $b$ of $f_{2} \cdots f_{n}$. Hence we have $a_{1} f_{2} \cdots f_{n} \in r_{R[x]}(I[x])$. Thus $\left(a_{1} f_{2}\right) f_{3} \cdots f_{n} \in r_{R[x]}(I[x])$. Since $a_{1} a_{2}$ is a coefficient of $a_{1} f_{2}$, we have $\left(a_{1} a_{2} c\right) \in r_{R}(I)$ for each coefficient $c$ of $f_{3} \cdots f_{n}$. Hence $a_{1} a_{2} f_{3} f_{4} \cdots f_{n} \in r_{R[x]}(I[x])$. Continuing, we see that $a_{1} \cdots a_{n} \in r_{R}(I)$

In the following proposition we show that if $I$ is an Armendariz left ideal of $R$, then $I[x]$ is an Armendariz left ideal of $R[x]$. In our proof of the following proposition, we use the method described in the proof of Theorem 2 of [1].

Proposition 2.3. Let $I$ be a left ideal of $R$. If $I$ is an Armendariz left ideal of $R$, then $I[x]$ is an Armendariz left ideal of $R[x]$.

Proof. Suppose that $I$ is an Armendariz left ideal of $R$ and let $f(t), g(t) \in$ $R[x][t]$ with $f . g \in r_{R[x][t]}(I[x][t])$. Write $f(t)=f_{0}+f_{1} t+\cdots+f_{n} t^{n}$ and $g(t)=g_{0}+g_{1} t+\cdots+g_{m} t^{m}$ where $f_{i}, g_{j} \in R[x]$. We need to prove each $f_{i} g_{j} \in r_{R[x]}(I[x])$. Let $k=\operatorname{deg} f_{0}+\cdots+\operatorname{deg} f_{n}+\operatorname{deg} g_{0}+\cdots+\operatorname{deg} g_{m}$, where deg denotes the $x$-degree and the degree of the zero polynomial is taken to be 0 . Then $f\left(x^{k}\right)=f_{0}+f_{1} x^{k}+\cdots+f_{n} x^{k n}, g\left(x^{k}\right)=g_{0}+g_{1} x+\cdots+g_{m} x^{k m} \in R[x]$ and the set of coefficients of the $f_{i}$ 's (resp., $g_{i}$ 's) equals the set of coefficients of $f\left(x^{k}\right)$ (resp., $\left.g\left(x^{k}\right)\right)$. Since $f(t) g(t) \in r_{R[x][t]}(I[x][t])$ and $x$ commutes with elements of $R, f\left(x^{k}\right) g\left(x^{k}\right) \in r_{R[x][t]}(I[x][t])$, we have $f\left(x^{k}\right) g\left(x^{k}\right) \in r_{R[x]}(I[x])$. Since $I$ is an Armendariz left ideal of $R$, each coefficient of $f_{i} g_{j}$ annihilates $I$. Thus $f_{i} g_{j} \in r_{R[x]}(I[x])$.

Next, we show that every Armendariz left ideal is an abelian left ideal. In our proof of the following proposition, we employ the methods used in the proofs of Lemma 7 and Corollary 8 of [4].

Proposition 2.4. If $I$ is an Armendariz left ideal of $R$, then we have the following assertions: 
(1) If $a b \in r_{R}(I), a c^{n} b \in r_{R}(I)$ for some $a, b, c$ of $R$ and some integer $n \geq 1$, then $a c b \in r_{R}(I)$.

(2) If $a b \in r_{R}(I)$ and $c^{n}$ is central for some $a, b, c$ of $R$ and some integer $n \geq 1$, then $a c b \in r_{R}(I)$.

Proof. (1) Consider $f(x)=a(1-c x), g(x)=\left(1+c x+\cdots+c^{n-1} x^{n-1}\right) b \in R[x]$. Then $f(x) g(x) \in r_{R[x]}\left(I[x]\right.$ and so $a b c \in r_{R}(I)$, because $I$ is an Armendariz left ideal.

(2) follows from (1) since $a c^{n} b \in r_{R}(I)$.

Corollary 2.5. If $I$ is an Armendariz left ideal of $R$, then $I$ is an abelian left ideal.

Proof. Assume that $I$ is an Armendariz left ideal. Consider $e=e^{2} \in R$ and let $a=e, b=(1-e), c=e r(1-e)$ with $r \in R$. Then clearly $a b \in r_{R}(I)$ and $e^{2}=0$, hence $a c b \in r_{R}(I)$ by Proposition 2.4. Letting $a_{1}=1-e, b_{1}=e$ and $c_{1}=(1-e) r e$, we also have $a_{1} c_{1} b_{1} \in r_{R}(I)$ similarly. Thus er $-r e \in r_{R}(I)$.

We know that any subring of an Armendariz ring is Armendariz. In the following proposition we prove that any left ideal of $R$ is an Armendariz left ideal provided that $R$ is Armendariz.

Proposition 2.6. If $R$ is a Armendariz ring, then each left ideal of $R$ is an Armendariz left ideal.

Proof. Let $R$ be an Armendariz ring and $I$ be a left ideal of $R$. If $f(x)=$ $\sum_{i=0}^{n} a_{i} x^{i}, g(x)=\sum_{j=0}^{n} b_{j} x^{j}$ are elements of $R[x]$ such that

$$
f(x) g(x) \in r_{R[x]}(I[x]),
$$

then $f(x) g(x) \in r_{R}(I)[x]$. Thus we have $d f(x) g(x)=0$ for any $d \in I$. Since $R$ is Armendariz, $d a_{i} b_{j}=0$ for all $i, j$. Thus $a_{i} b_{j} \in r_{R}(I)$.

It is obvious that the converse of Proposition 2.6 is true, because $R$ is an ideal of $R$ and $r_{R}(R)=0$.

Let $R$ be a ring and $M$ be an $(R, R)$-bimodule. The trivial extension of $R$ by $M$ is defined to be the $\operatorname{ring} T(R, M)=R \oplus M$ with the usual addition and the multiplication $\left(r_{1}, m_{1}\right)\left(r_{2}, m_{2}\right)=\left(r_{1} r_{2}, r_{1} m_{2}+m_{1} r_{2}\right)$. We know that $T(R, M)$ is isomorphic to the ring of all matrices $\left(\begin{array}{cc}r & m \\ 0 & r\end{array}\right)$ where $r \in R, m \in M$ and usual matrix operations are used. It is proved in [6] that $R$ is reduced ring if and only if the trivial extension $T(R, R)$ is an Armendariz ring. Next we give an example of a nonzero Armendariz left ideal of a non-Armendariz ring.

Example 2.7. Let $R=\mathbb{Z}_{4}$ and $S=T(R, R)$. Since the ring $R$ is not reduced, $S$ is not Armendariz. Write $a=\left(\begin{array}{ll}0 & \overline{2} \\ 0 & 0\end{array}\right), I=S a$ and $r(I)=r_{S}(I)$, then

$$
r(I)=\left\{\left(\begin{array}{cc}
r & b \\
0 & r
\end{array}\right) \mid r \in\{0, \overline{2}\}, b \in \mathbb{Z}_{4}\right\} .
$$

Since $r(I)$ is an ideal of $\mathrm{S}$ and $S / r(I)$ is a reduced ring, $I$ is an Armendariz left ideal of $S$. 
A ring $R$ is called right Ore if given $a, b \in R$ with $b$ regular there exist $a_{1}, b_{1} \in R$ with $b_{1}$ regular such that $a b_{1}=b a_{1}$. It is a well-known fact that $R$ is a right Ore ring if and only if there exists a classical right quotient ring of $R$.

Theorem 2.8. Suppose that there exists a classical right quotient ring $Q$ of a ring $R$. If $I$ is an Armendariz left ideal of $R$, then $Q I$ is an Armendariz left ideal of $Q$.

Proof. We employ the methods used in the proofs of Theorem 16 of [5] and Theorem 12 of [4]. Consider $f(x)=\sum_{i=0}^{m} \alpha_{i} x^{i}, g(x)=\sum_{j=0}^{n} \beta_{j} x^{j} \in Q[x]$ such that $f(x) g(x) \in r_{Q[x]}(Q I[x])$. We may assume that $\alpha_{i}=a_{i} u^{-1}, \beta_{j}=b_{j} v^{-1}$ with $a_{i}, b_{j} \in R$ for all $i, j$ and regular elements $u, v \in R$. For each $j$ there exists $c_{j} \in R$ and regular element $w \in R$ such that $u^{-1} b_{j}=c_{j} w^{-1}$.

Put $f_{1}(x)=\sum_{i=0}^{m} a_{i} x^{i}, g_{1}(x)=\sum_{j=0}^{n} c_{j} x^{j} \in R[x]$. Then we have

$$
\begin{aligned}
f(x) g(x) & =\sum_{i=0}^{m} \sum_{j=0}^{n} \alpha_{i} \beta_{j} x^{i+j} \\
& =\sum_{i=0}^{m} \sum_{j=0}^{n} a_{i}\left(u^{-1} b_{j}\right) v^{-1} x^{i+j} \\
& =\sum_{i=0}^{m} \sum_{j=0}^{n} a_{i} c_{j}(v w)^{-1} x^{i+j}=f_{1}(x) g_{1}(x)(u w)^{-1}
\end{aligned}
$$

hence $f_{1}(x) g_{1}(x) \in r_{R[x]}(I[x])$. Since the left ideal $I$ of $R$ is Armendariz, $a_{i} c_{j} \in r_{R}(I)$ for all $i, j$ and so $\alpha_{i} \beta_{j}=a_{i} u^{-1} b_{j} v^{-1}=a_{i} c_{j} w^{-1} u^{-1} \in r_{Q}(Q I)$ for all $i, j$. Therefore $Q I$ is an Armendariz left ideal of $Q$.

\section{Annihilator ideals with IFP}

Following Bell [3], a one-sided ideal $J$ of a ring $R$ is said to have the insertionof-factors property in $R$ (or, briefly, we say $J$ has the IFP in $R$ ) if, for elements $a, b$ of $R$ the condition $a b \in J$ implies $a R b \subseteq J$. We also say $J$ is an ideal with IFP in this case. Clearly $0=r_{R}(R)$ is an ideal with IFP if and only if $R$ is an IFP ring (These rings have also been studied under the names semicommutative rings and zero-insertive (ZI) rings in the literature). In this section we record a couple of results involving the condition ' $r_{R}(I)$ is with IFP'.

Proposition 3.1. Suppose that $I$ is a one-sided ideal of a ring $R$ and $\Delta$ is a multiplicatively closed subset of $R$ consisting of central regular elements. If $r_{R}(I)$ has the IFP in $R$, then $r_{\Delta^{-1} R}\left(\Delta^{-1} I\right)$ has the IFP in $\Delta^{-1} R$.

Proof. We employ the method used in the proof of Proposition 3.1 of [4]. Let $\alpha \beta \in r_{\Delta^{-1} R}\left(\Delta^{-1} I\right)$ with $\alpha=u^{-1} a, \beta=v^{-1} b, u, v \in \Delta$ and $a, b \in R$. Since $\Delta$ is contained in the center of $R$, we have $0=\Delta^{-1} I \alpha \beta=\Delta^{-1} I u^{-1} a v^{-1} b=$ $\Delta^{-1} \operatorname{Iab}(u v)^{-1}$ so $I a b=0$. It follows that $a r b \in r_{R}(I)$ for all $r \in R$, since $r_{R}(I)$ has the IFP. Now for $\gamma=w^{-1} r$ with $w \in \Delta$ and $r \in R, \Delta^{-1} I \alpha \gamma \beta=$ $\Delta^{-1} \operatorname{Iarb}(u w v)^{-1}=0$. Hence $r_{\Delta^{-1} R}\left(\Delta^{-1} I\right)$ has the IFP. 
A ring $R$ is called locally finite if every finite subset of $R$ generates a finite semigroup multiplicatively. Finite rings are clearly locally finite and the algebraic closure of a finite field is locally finite but is not finite.

In our proof of the following proposition, we employ the method used in the proof of Proposition 16 of [4].

Proposition 3.2. Let $R$ be a locally finite ring and let $I$ be an Armendariz left ideal of $R$. Then $r_{R}(I)$ has the IFP.

Proof. Let $a b \in r_{R}(I)$ with $a, b \in R$. For any $r \in R$ since $R$ is locally finite there exist integers $m, k \geq 1$ such that $r^{m}=r^{m+k}$. So we obtain inductively $r^{m}=r^{m} r^{k}=r^{m} r^{2 k}=\cdots=r^{m} r^{m k}=r^{m(k+1)}$, put $h=k+1$ then $r^{m}=$ $\left(r^{m}\right)^{h}$ with $h \geq 2$. Notice that $r^{(h-1) m}=r^{(h-2) m} r^{m}=r^{(h-2) m}\left(r^{m}\right)^{h}=$ $r^{2(h-1) m}=\left(r^{(h-1) m}\right)^{2}$. Whence $r^{(h-1) m}$ is an idempotent and so by Corollary 2.5, $a r^{(h-1) m}-r^{(h-1) m} a \in r_{R}(I)$ and $a b r^{(h-1) m}-r^{(h-1) m} a b \in r_{R}(I)$. Thus $r^{(h-1) m} a b \in r_{R}(I)$. On the other hand by Corollary 2.5, $a r^{(h-1) m}-r^{(h-1) m_{a}} a \in$ $r_{R}(I)$, so $a r^{(h-1) m} b-r^{(h-1) m} a b \in r_{R}(I)$, hence $a r^{(h-1) m} b \in r_{R}(I)$. So by Proposition 2.4, arb $\in r_{R}(I)$ for all $r \in R$.

Acknowledgment. The authors are deeply indebted to the referee for many helpful comments and suggestions for the improvement of this article.

\section{References}

[1] D. D. Anderson and V. Camillo, Armendariz rings and Gaussian rings, Comm. Algebra 26 (1998), no. 7, 2265-2272.

[2] E. P. Armendariz, A note on extensions of Baer and P.P.-rings, J. Aust. Math. Soc. 18 (1974), 470-473.

[3] H. E. Bell, Near-rings in which each element is a power of itself, Bull. Aust. Math. Soc. 2 (1970), 363-368.

[4] C. Huh, Y. Lee, and A. Smoktunowicz, Armendariz rings and semicommutative rings, Comm. Algebra 30 (2002), no. 2, 751-761.

[5] N. K. Kim and Y. Lee, Armendariz rings and reduced rings, J. Algebra 223 (2000), no. 2, 477-488.

[6] T. K. Lee and T. L. Wong, On Armendariz rings, Houston J. Math. 29 (2003), no. 3, 583-593.

[7] M. B. Rege and S. Chhawchharia, Armendariz rings, Proc. Japan Acad. Ser. A Math. Sci. 73 (1997), no. 1, 14-17.

[8] G. Shin, Prime ideals and sheaf representation of a pseudo symmetric ring, Trans. Amer. Math. Soc. 184 (1973), 43-60.

Sh. Ghalandarzadeh

Department of Mathematics

K. N. Toosi University of Technology

TEHRAN, IRAN

E-mail address: ghalandarzadeh@kntu.ac.ir 
H. HaJ Seyyed Javadi

Department of Mathematics

Shahed University

TEHRAN, Iran

E-mail address: h.s.javadi@shahed.ac.ir

M. KHORAMDEL

Department of Mathematics

K. N. Toosi University of Technology

TEHRAN, IRAN

E-mail address: mehdikhoramdel@Gmail.com

M. Shamsaddini FARD

Department of Mathematics

K. N. Toosi University of Technology

TEHRAN, IRAN 